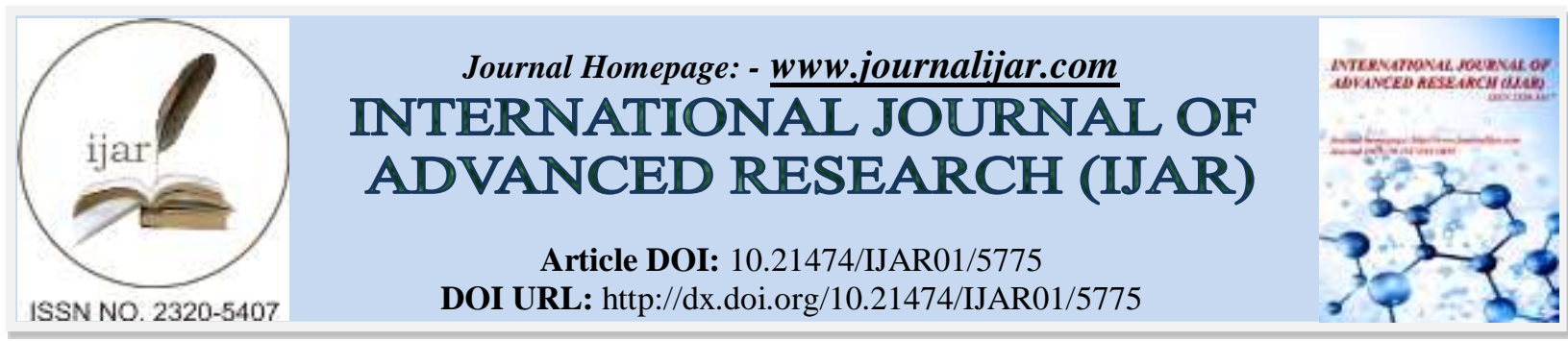

RESEARCH ARTICLE

\title{
PERIODOGRAM ANALYSIS WITH MISSED OBSERVATION BETWEEN TWO VECTOR VALUED STOCHASTIC PROCESS.
}

\begin{abstract}
M. A. Ghazal ${ }^{1}$, A. I. El-desokey ${ }^{2}$ and A. M. Ben Aros ${ }^{3}$.
1. Department of Mathematics, Faculty of Science, University of Damietta, A. R. Egypt.

2. Lecturer in Higher Future Institute for Specialized Technological Studies, Egypt.

3. Department of Mathematics, Faculty of Science, University of Damietta, Egypt.
\end{abstract}

\section{Manuscript Info}

Manuscript History

Received: 05 September 2017

Final Accepted: 07 October 2017

Published: November 2017

Key words:-

Modified periodogram, Spectral density,

Spectral measure, Continuous Stability

time series, Missing values, Data

window.

\section{Abstract}

The estimation of the spectral measure, covariance and spectral density functions strictly stability $(\mathrm{r}+\mathrm{s})$ vector-valued time series are considered, under the assumption that some of observations are missed. The modified periodograms with missed observation are calculated. This method is applied in the climate.

Copy Right, IJAR, 2017,. All rights reserved.

\section{Introduction:-}

Several authors discussed the properties of the smoothing periodograms using data window and considered the estimation of spectral measure of stationary process, Brillinger(1969), Dahlhaus(1985), Ghazal and Farag(2000), Teama and Bakouch(2004), Ghazal(2001,2005), Ghazal, Faraj and El-Desokey(2005), Ghazal and Elhassanein(2006), Ghazal, Mokaddis and El- Desokey(2010), Elhassanein(2013).

$$
\text { Let } \quad B(t)=\left[\begin{array}{c}
X(t) \\
Y(t)
\end{array}\right], t=0, \pm 1, \pm 2, \ldots . .
$$

with $X(t), \quad r$ vector-valued and $Y(t), \quad s$ vector-valued a strictly stability $(r+s)$ series, where $Y(t) \approx \sum a(t-u) X(t)$. We construct the statistics $I_{W W}{ }^{(T)}(\lambda)(-\infty<\lambda<\infty)$, the matrix of second order smoothing modified periodograms, $F_{B B}{ }^{(T)}(\lambda)$, which is the matrix of second order spectral measures, and $f_{B B}{ }^{(T)}(\lambda)$ is the matrix of second order spectral densities .

Suppose that :

$$
\begin{aligned}
& E\left\{\left[X(t+u)-C_{x}\right]\left[X(t)-C_{x}\right]^{T}\right\}=C_{x x}(u), \\
& E\left\{\left[X(t+u)-C_{x}\right]\left[Y(t)-C_{y}\right]^{T}\right\}=C_{x y}(u), \\
& E\left\{\left[Y(t+u)-C_{y}\right]\left[Y(t)-C_{y}\right]^{T}\right\}=C_{y y}(u),
\end{aligned}
$$


and we defined the second-order spectral densities by

$$
\begin{aligned}
& f_{x x}(\lambda)=(2 \pi)^{-1} \int_{-\infty}^{\infty} C_{x x}(u) \operatorname{Exp}(-i \lambda u) d u, \\
& f_{x y}(\lambda)=(2 \pi)^{-1} \int_{-\infty}^{\infty} C_{x y}(u) \operatorname{Exp}(-i \lambda u) d u, \quad \text { for } \lambda \in R \\
& f_{y y}(\lambda)=(2 \pi)^{-1} \int_{-\infty}^{\infty} C_{y y}(u) \operatorname{Exp}(-i \lambda u) d u,
\end{aligned}
$$

we defined the second-order spectral measures by

$$
\begin{array}{ll}
F_{x x}(\lambda)=\int_{0}^{\lambda} f_{x x}(\alpha) d \alpha, & (0<\lambda<\pi) \\
F_{x y}(\lambda)=\int_{0}^{\lambda} f_{x y}(\alpha) d \alpha, & (0<\lambda<\pi) \\
F_{y y}(\lambda)=\int_{0}^{\lambda} f_{y y}(u) d \alpha, & (0<\lambda<\pi)
\end{array}
$$

we construct estimates $C_{X X}{ }^{(T)}(u), C_{X Y}{ }^{(T)}(u), C_{Y Y}{ }^{(T)}(u), f_{X X}{ }^{(T)}(\lambda), f_{X Y}{ }^{(T)}(\lambda), f_{Y Y}{ }^{(T)}(\lambda), F_{X X}{ }^{(T)}(\lambda)$, $F_{X Y}{ }^{(T)}(\lambda)$ and $F_{Y Y}{ }^{(T)}(\lambda)$. These estimates based on the matrix of second order smoothing modified periodograms .

\section{Assumption I.}

Let $X(t)$ is a strictly stability continuous time series all of whose moments are exist. For each $j=1,2, \ldots, k-1$ and any k-tuple $a_{1}, a_{2}, \ldots, a_{k}$ we have,

$$
\int_{R^{K-1}}\left|u_{j} \| C_{a_{1}, \ldots, a_{k}}\left(u_{1}, \ldots ., u_{k-1}\right)\right| d_{t_{1}} \ldots d_{t_{K-1}}<\infty, \quad k=2,3, \ldots
$$

where

$$
\begin{gathered}
C_{a_{1}, \ldots, a_{k}}\left(u_{1}, \ldots, u_{k-1}\right)=\operatorname{cum}\left\{X_{a_{1}}\left(t+u_{1}\right), X_{a_{1}}\left(t+u_{2}\right), \ldots, X_{a_{k}}(t)\right\}, \\
\left(a_{1}, \ldots, a_{k}=1,2, \ldots, r, \quad u_{1}, \ldots, u_{k-1}, \quad t \in R, k=2,3, \ldots .\right)
\end{gathered}
$$

\section{Assumption II.}

Let $\Psi(\alpha), \alpha \in R$ is a weight function which is bounded and has bounded first derivative such that

$$
\int_{-\pi}^{\pi} \Psi(\alpha) d \alpha=1
$$

Given $B_{T}>0$ we then set

$$
\Psi^{(T)}(\alpha)=B_{T}^{-1} \Psi^{(T)}\left(B_{T}^{-1} \alpha\right)
$$

\section{Assumption III.}

Let $h_{a}^{(T)}(t)$ is bounded has bounded variation and vanishes for $0<t<T-1$, is called data window and satisfies 


$$
\begin{gathered}
\frac{1}{T} \int_{0}^{T} h_{a}{ }^{(T)} d_{t} \underset{T \rightarrow \infty}{\longrightarrow} \int_{0}^{1} h_{a}(u) d u, \quad a=\overline{1, r} \\
G^{(T)}{ }_{a_{1}, \ldots, a_{k}(\lambda)}=\int_{0}^{T}\left[\prod_{j=1}^{k} h_{a_{j}}^{(T)}(t)\right] \exp \{-i \lambda t\} d t, \\
\text { for }-\infty<\lambda<\infty, a_{1}, \ldots, a_{k}=1, \ldots, r
\end{gathered}
$$

Let $H_{a}(t), a=1,2, \ldots, r(t \in R)$ be a process independent of $B(t)$ such that for every $t$,

$$
\begin{aligned}
& P\left[H_{a}(t)=1\right]=p_{a}, \\
& P\left[H_{a}(t)=0\right]=q_{a} .
\end{aligned}
$$$$
\text { Note that } E\left\{H_{a}(t)\right\}=P \text {. }
$$

The success of recording an observation not depends on the fail of another and so they are independent . We may then define the modified series as

$$
W(t)=H(t) B(t)
$$

where

$$
W_{a}(t)=H_{a}(t) B_{a}(t)
$$

and

$$
H_{a}(t)=\left\{\begin{array}{ll}
1, & \text { if } X_{a}(t), Y_{a}(t) \text { are observed } \\
0, & \text { otherwise }
\end{array},\right.
$$

We construct the expanded finite Fourier transform with data window with missed observations as :

$$
d_{a}^{(T)}(\lambda)=\left[2 \pi \int_{0}^{T}\left(h_{a}^{(T)}(t)\right)^{2}\right]^{-1 / 2} \int_{-\infty}^{\infty} h_{a}^{(T)}(t) W_{a}(t) \exp \{-i \lambda t\} d t, \quad \text { for } \lambda \in R,
$$

The paper is organized as follows : In Section(1) Introduction, Section (2) we will considered the smoothing modified periodograms, We will study the statistical properties of the spectral measure and spectral density in Section (3), application on our theoretical study in the climate in Section (4).

\section{The Smoothing Modified Periodograms.}

In this section, the modified periodogram will be constructed. Using expanded finite Fourier transform (1.10) we construct the modified periodogram as:

$$
I_{a b}{ }^{(T)}(\lambda)=\left\{2 \pi G_{a b}{ }^{(T)}(0)\right\}^{-1} \partial_{a}^{(T)}(\lambda) \overline{\partial_{b}{ }^{(T)}(\lambda)},
$$

where

$$
\partial_{X_{a}}^{(T)}(\lambda)=\int_{0}^{T} h_{a}(t) W_{a}(t) \exp (-i \lambda t) d t
$$

The bar denotes the complex conjugate. The moments of modified periodogram will be given in the following theorems.

\section{Theorem 2.1}

Let $W_{a}(t)=H_{a}(t) B_{a}(t), a=1,2, \ldots ., \min (r, s)$ are missed observations on the strictly stability continuous series which satisfies Assumption I with mean zero, $h_{a}(u),-\infty<u<\infty$ satisfies Assumption (III) for $a=1, \ldots, \min (r, s)$, and let 


$$
I_{W W}{ }^{(T)}(\lambda)=\left[I_{a b}{ }^{(T)}(\lambda)\right]=\left[\left\{2 \pi G_{a b}{ }^{(T)}(0)\right\}^{-1} \partial_{a}{ }^{(T)}(\lambda) \overline{\partial_{b}{ }^{(T)}(\lambda)}\right],
$$

then

$$
E\left[I_{a b}{ }^{(T)}(\lambda)\right]=\left[\begin{array}{cc}
P_{a_{1} a_{2}} f_{a_{1} a_{2}}(\lambda) & P_{a_{1} b_{2}} f_{a_{1} b_{2}}(\lambda) A(\lambda)^{T} \\
P_{b_{1} a_{2}} A(\lambda) f_{b_{1} a_{2}}(\lambda) & P_{b_{1} b_{2}} A(\lambda) f_{b_{1} b_{2}}(\lambda) A(\lambda)^{T}
\end{array}\right]+\left[\begin{array}{cc}
O\left(T^{-1}\right) & O\left(T^{-1}\right) \\
O\left(T^{-1}\right) & O\left(T^{-1}\right)
\end{array}\right],
$$

Where $O\left(T^{-1}\right)$ is uniform in $\lambda$ and $A(\lambda)=f_{y x}(\lambda) f_{x x}(\lambda)^{-1}$.

and

$$
\begin{aligned}
& \operatorname{Cov}\left[I_{a_{1} b_{1}}{ }^{(T)}(\lambda), I_{a_{2} b_{2}}{ }^{(T)}(\mu)\right]=\left\{G_{a_{1} b_{1}}{ }^{(T)}(0) G_{a_{2} b_{2}}{ }^{(T)}(0)\right\}^{-1} \times \\
& \times\left[\left(P^{4} G_{a_{1} a_{2}}(\lambda-\mu) \overline{G_{b_{1} b_{2}}(\lambda-\mu)} V Z+P^{4} G_{a_{1} b_{2}}(\lambda+\mu) \overline{G_{b_{1} a_{2}}(\lambda+\mu)} V Z\right]+\right. \\
& +T^{-2} M_{a_{1} b_{1} a_{2} b_{2}}^{(T)}(\lambda, \mu)+O\left(T^{-1}\right),
\end{aligned}
$$

where

$$
\begin{gathered}
V=\left[\begin{array}{cc}
f_{a_{1} a_{2}}(\lambda) & f_{a_{1} b_{2}}(\lambda) A(\lambda)^{T} \\
A(\lambda) f_{b_{1} a_{2}}(\lambda) & A(\lambda) f_{b_{1} b_{2}}(\lambda) A(\lambda)^{T}
\end{array}\right], Z=\left[\begin{array}{cc}
f_{a_{1} a_{2}}(-\lambda) & f_{a_{1} b_{2}}(-\lambda) A(\lambda)^{T} \\
A(\lambda) f_{b_{1} a_{2}}(-\lambda) & A(\lambda) f_{b_{1} b_{2}}(-\lambda) A(\lambda)^{T}
\end{array}\right], \\
O=\left[\begin{array}{ll}
O(1) & O(1) \\
O(1) & O(1)
\end{array}\right]
\end{gathered}
$$

\section{Lemma 2.1.}

Let $h_{a}{ }^{(T)}(t), t \in R, a=1, \ldots, \min (r, s)$ is bounded by a constant $L$ and satisfying

$$
\left|h_{a}^{(T)}(t+u)-h_{a}(t)\right| \leq C|u|
$$

then

$$
\left|\int_{0}^{T} h_{a_{1}}{ }^{(T)}(t) h_{a_{2}}{ }^{(T)}(t) \exp (-i \lambda t) d t\right| \leq \frac{1}{|\lambda / 2|}+L C,
$$

for some constants $L, C$ and $\lambda, \lambda \in R, \lambda \neq 0, a_{1}, a_{2}=1, \ldots, \min (r, s)$.

\section{Corollary 2.1.}

Under the conditions of theorem (2.1) we have

$$
\begin{gathered}
E\left[I_{a b}{ }^{(T)}(\lambda)\right] \rightarrow P^{2}\left[\begin{array}{cc}
f_{a_{1} a_{2}}(\lambda) & f_{a_{1} b_{2}}(\lambda) A(\lambda)^{T} \\
A(\lambda) f_{b_{1} a_{2}}(\lambda) & A(\lambda) f_{b_{1} b_{2}}(\lambda) A(\lambda)^{T}
\end{array}\right] \text { as } T \rightarrow \infty \\
a, b=1, \ldots, \min (r, s), \lambda \in R .
\end{gathered}
$$

Proof

The prove comes directly from (2.3) by taking the limits for both sides and then using the given conditions . 


\section{Corollary 2.2.}

Under the conditions of theorem (2.1) then for all $\lambda, \mu \in R$,

$$
\begin{aligned}
\left.\lim _{T \rightarrow \infty} \operatorname{Cov} \mid I_{a_{1} b_{1}}{ }^{(T)}(\lambda), I_{a_{2} b_{2}}{ }^{(T)}(\mu)\right] & = \\
& = \begin{cases}P^{4} \delta(\lambda-\mu) V Z+P^{4} \delta(\lambda+\mu) V Z, & \text { if } \lambda \pm \mu=0 \\
0, & \text { if } \lambda \pm \mu \neq 0\end{cases}
\end{aligned}
$$

Where $\delta(\lambda-\mu)$ is the Kroncker delta function which is given by :

\section{Proof:}

$$
\delta(\lambda)= \begin{cases}1, & \lambda=0 \\ 0, & \text { ow }\end{cases}
$$

When $\lambda \pm \mu=0$, and by using the Assumption III then we get from (2.4)

$$
\operatorname{Cov}\left[I_{a_{1} b_{1}}{ }^{(T)}(\lambda), I_{a_{2} b_{2}}{ }^{(T)}(\mu)\right]=P^{4} \delta(\lambda-\mu) V Z+P^{4} \delta(\lambda+\mu) V Z+O\left(T^{-1}\right)
$$

In the limit, then

$$
\operatorname{Cov}\left[I_{a_{1} b_{1}}{ }^{(T)}(\lambda), I_{a_{2} b_{2}}{ }^{(T)}(\mu)\right]=P^{4} \delta(\lambda-\mu) V Z+P^{4} \delta(\lambda+\mu) V Z .
$$

Now, when $\lambda \pm \mu \neq 0, \lambda, \mu \in R$, then take the modulus for both sides of (2.4) and then using lemma (2.1) and the boundedness of $f_{a b}(\lambda) a, b=1,2, \ldots, \min (r, s), \lambda \in R$, we obtain

$$
\begin{aligned}
\mid \operatorname{Cov}\left[I_{a_{1} b_{1}}{ }^{(T)}(\lambda), I_{a_{2} b_{2}}{ }^{(T)}(\mu)\right] \leq & \left\{G_{a_{1} b_{1}}{ }^{(T)}(0) G_{a_{2} b_{2}}{ }^{(T)}(0)\right\}^{-1} \times \\
& \times\left\{\left[\frac{2 L_{1} v_{1}}{|\sin (\lambda+\mu) / 2|}\right]^{2} K^{2}+\left[\frac{2 L_{2} v_{2}}{|\sin (\lambda-\mu) / 2|}\right]^{2} K^{2}\right\}+ \\
& +T^{-2}\left|T^{-2} M_{a_{1} b_{1} a_{2} b_{2}}^{(T)}(\lambda, \mu)\right|+\left(T^{-1}\right),
\end{aligned}
$$

where, for some constant $K$, we have

$$
\begin{aligned}
\left|T^{-2} M_{a_{1} b_{1} a_{2} b_{2}}^{(T)}(\lambda, \mu)\right| \leq & K\left\{\left[\frac{2 L_{1} v_{1}}{|\sin (\lambda+\mu) / 2|}\right]+\left[\frac{2 L_{2} v_{2}}{|\sin (\lambda-\mu) / 2|}\right] \times\right. \\
& \left.\times\left[\frac{2 L_{3} v_{3}}{|\sin (\lambda+\mu) / 2|}\right]+\left[\frac{2 L_{4} v_{4}}{|\sin (\lambda-\mu) / 2|}\right]\right\},
\end{aligned}
$$

using lemma (2.1) we get $\operatorname{Cov}\left[I_{a_{1} b_{1}}{ }^{(T)}(\lambda), I_{a_{2} b_{2}}{ }^{(T)}(\mu)\right] \rightarrow 0$ as $T \rightarrow \infty$.hence, the corollary is obtained. In the case of $\lambda= \pm \mu$ corollary (2.2) indicates corollary (2.3) as the following .

\section{Corollary 2.3.}

Under the conditions of theorem(2.1) and corollary (2.2) then,

$$
\lim _{T \rightarrow \infty} D\left[I_{a b}{ }^{(T)}(\lambda)\right]=\left\{\begin{array}{lr}
P^{4} \delta(\lambda-\mu) V Z, & \text { if } \lambda=\mu=\omega \neq 0 \\
P^{4} \delta(\lambda-\mu) V Z+P^{4} \delta(\lambda+\mu) V Z, & \text { if } \lambda=\mu=\omega=0
\end{array}\right.
$$




\section{Proof}

By substituting about $\lambda=\mu=\omega, \omega \in R, \quad a_{1}=a_{2}=a, b_{1}=b_{2}=b, a, b=1, . ., \min (r, s)$ into corollary(2.2) we get

$$
\lim _{T \rightarrow \infty} D\left[I_{a b}^{(T)}(\lambda)\right]=P^{4} \delta(\omega-\omega) V Z+P^{4} \delta(\omega+\omega) V Z,
$$

when $\omega \neq 0$, by noting that $f_{a b}(\omega)=f_{b a}(-\omega)$ into $V, Z, a, b=1, \ldots, \min (r, s), \omega \in R$ then

$$
\lim _{T \rightarrow \infty} D\left[I_{a b}{ }^{(T)}(\lambda)\right]=P^{4} \delta(\omega-\omega) V Z .
$$

When $\omega=0$, then we obtain

$$
\lim _{T \rightarrow \infty} D\left[I_{a b}{ }^{(T)}(\lambda)\right]=P^{4} V Z+P^{4} V Z .
$$

Hence the proof is complete .

\section{Asymptotic moments of spectral measure and spectral density function}

In this section we will study the statistical properties of $F_{x x}{ }^{(T)}(\lambda)$ and $f_{x x}{ }^{(T)}(\lambda)$ by deriving mean and covariance. Let $f_{x x}{ }^{(T)}(\lambda)$ be defined as (1.3) and $F_{x x}{ }^{(T)}(\lambda)$ be defined as (1.4), then from Theorem (2.1) we get,

\section{Theorem 3.1}

Let $W(t)$ satisfies Assumption $I$ then:

$$
\begin{aligned}
E\left\{F_{a b}{ }^{(T)}(\lambda)\right\} & =P^{2}\left[\begin{array}{cc}
F_{a_{1} a_{2}}(\lambda) & F_{a_{1} b_{2}}(\lambda) A(\lambda)^{T} \\
A(\lambda) F_{b_{1} a_{2}}(\lambda) & A(\lambda) F_{b_{1} b_{2}}(\lambda) A(\lambda)^{T}
\end{array}\right]+ \\
& +\left[\begin{array}{cc}
O\left(T^{-1}\right) & O\left(T^{-1}\right) \\
O\left(T^{-1}\right) & O\left(T^{-1}\right)
\end{array}\right],
\end{aligned}
$$

where $O\left(T^{-1}\right)$ is uniform in $\lambda$.

$$
\begin{aligned}
\operatorname{cov}\left\{F_{a_{1} b_{1}}{ }^{(T)}\left(\lambda_{1}\right), F_{a_{2} b_{2}}{ }^{(T)}\left(\lambda_{2}\right)\right\} & =\left[\left\{G_{a_{1} b_{1}}{ }^{(T)}(0) G_{a_{2} b_{2}}{ }^{(T)}(0)\right\}^{-1}\right] \times \\
& \times P^{4} G_{a_{1} a_{2} b_{1} b_{2}}(0)\left[\int_{-\infty}^{\lambda_{1}} V Z d \alpha_{1}+\int_{-\infty}^{\lambda_{1}} V Z d \alpha_{1}\right]+O\left(T^{-1}\right)
\end{aligned}
$$

\section{Corollary 3.1.}

Let $W_{a}(t)=H_{a}(t) B_{a}(t), a=1,2, \ldots, \min (r, s)$ are missed observations on the strictly stability continuous series which satisfies Assumption(I) with mean zero, $h_{a}(t),-\infty<t<\infty$, be data window satisfies Assumption (III) for $a=1, \ldots, \min (r, s)$, and let

then

$$
I_{w w}{ }^{(T)}(\lambda)=\left[I_{a b}{ }^{(T)}(\lambda)\right]=\left[\left\{2 \pi G_{a b}{ }^{(T)}(0)\right\}^{-1} d_{a}^{(T)}(\lambda) \overline{d_{b}^{(T)}(\lambda)}\right]
$$

$$
E\left[F_{a b}{ }^{(T)}(\lambda)\right] \underset{T \rightarrow \infty}{\rightarrow} P^{2}\left[\begin{array}{cc}
F_{a_{1} a_{2}}(\lambda) & F_{a_{1} b_{2}}(\lambda) A(\lambda)^{T} \\
A(\lambda) F_{b_{1} a_{2}}(\lambda) & A(\lambda) F_{b_{1} b_{2}}(\lambda) A(\lambda)^{T}
\end{array}\right],
$$

for all $a, b=1, \ldots, \min (r, s)$. 


\section{proof}

Formula (3.3) comes directly by taking the limits for both sides of (3.1) and the proof is complete.

\section{Corollary 3.2.}

Under the conditions of theorem (3.1) if the spectral density function $f_{a b}(x)$ is bounded by a constant $k$, $a, b=1, \ldots, \min (r, s)$ and continuous at point $x=\lambda, \lambda \in R$, then

$$
\lim _{T \rightarrow \infty} \operatorname{Cov}\left[F_{a_{1} b_{1}}{ }^{(T)}(\lambda), F_{a_{2} b_{2}}{ }^{(T)}(\mu)\right]=0,
$$

for $a_{j}, b_{j}=1, \ldots, \min (r, s), j=1, \ldots, k, k=1,2, \ldots$

\section{Proof}

Taking the modulus on both sides of (3.2), we get

$$
\begin{gathered}
\left|\operatorname{Cov}\left[F_{a_{1} b_{1}}{ }^{(T)}(\lambda), F_{a_{2} b_{2}}{ }^{(T)}(\mu)\right] \leq(2 \pi)\right| p^{4} \mid G_{a_{1} a_{2} b_{1} b_{2}}(0) \times \\
\times\left\{G_{a_{1} b_{1}}{ }^{(T)}(0) G_{a_{2} b_{2}}{ }^{(T)}(0)\right\}^{-1} \times\left|P^{4}\right| G_{a_{1} a_{2} b_{1} b_{2}}(0) \times\left[\int_{-\infty}^{\lambda_{1}}|V| Z\left|d \alpha_{1}+\int_{-\infty}^{\lambda_{1}}\right| V|| Z \mid d \alpha_{1}\right]+O\left(T^{-1}\right)
\end{gathered}
$$

Using Assumption (III) and the boundedness of $f_{a b}(\lambda), a, b=1, \ldots, \min (r, s), \lambda \in R$ we get

$$
\operatorname{Cov}\left[F_{a_{1} b_{1}}{ }^{(T)}(\lambda), F_{a_{2} b_{2}}{ }^{(T)}(\mu)\right]=O\left(T^{-1}\right) \underset{T \rightarrow \infty}{\longrightarrow}=0 \text {. }
$$

Then the corollary is obtained .

\section{Lemma 3.1.}

Let $h_{a}^{(T)}(t),-\infty<t<\infty$, be data window satisfies Assumption III for $a=1, \ldots, \min (r, s)$ then $h_{a}^{(T)}(t)$ satisfies the following properties

$$
\begin{aligned}
& \text { 1. } \int_{t_{2}=0}^{T} \Psi_{a_{2} b_{2}}{ }^{(T)}\left(\lambda_{2}-\alpha_{2}\right) G_{a_{1} a_{2}}(\lambda-\mu) d t_{2}=2 \pi \Psi_{a_{2} b_{2}}{ }^{(T)}\left(\lambda_{2}-\mu\right)+O(1), \\
& \text { 2. } \int_{t_{2}=0}^{T} \Psi_{a_{2} b_{2}}{ }^{(T)}\left(\lambda_{2}-\alpha_{2}\right) G_{a_{1} a_{2}}(\lambda+\mu) \overline{G_{b_{1} b_{2}}(\lambda+\mu)} d t_{2}= \\
& =2 \pi G_{a_{1} a_{2} b_{1} b_{2}}{ }^{(T)}(0) \Psi_{a_{2} b_{2}}{ }^{(T)}\left(\lambda_{2}-\alpha_{1}\right)+O\left(T^{-1}\right) .
\end{aligned}
$$

\section{Theorem 3.2}

Let $W_{a}(t)=B_{a}(t) H_{a}(t), t=0, \pm 1, \ldots ., a=1, \ldots, \min (r+s)$ are missed observations on the strictly stability continuous $B_{a}(t), a=1, \ldots, \min (r+s), t \in R$ which satisfies Assumption I with mean zero, $h_{a}(t), a=1, \ldots, \min (r+s), t \in R$ be data window satisfies Assumption III, and let

$$
f_{a b}{ }^{(T)}(\lambda)=\int_{t=0}^{T} \Psi^{(T)}(\lambda-\alpha) \mathrm{I}_{a b}{ }^{(T)}(\alpha) d t
$$

Where $\Psi^{(T)}(\lambda-\alpha)$ is weight function which is defined in assumption II. Then

$$
E\left\{f_{a b}{ }^{(T)}(\lambda)\right\}=P^{2} \int_{t=0}^{T} \Psi_{a b}{ }^{(T)}(\lambda-\alpha)\left[\begin{array}{cc}
f_{a_{1} a_{2}}(\alpha) & f_{a_{1} b_{2}}(\alpha) A(\alpha)^{T} \\
A(\alpha) f_{b_{1} a_{2}}(\alpha) & A(\alpha) f_{b_{1} b_{2}}(\alpha) A(\alpha)^{T}
\end{array}\right] d t+
$$




$$
+\left[\begin{array}{ll}
O\left(T^{-1}\right) & O\left(T^{-1}\right) \\
O\left(T^{-1}\right) & O\left(T^{-1}\right)
\end{array}\right]
$$

and

$$
\begin{gathered}
\operatorname{cov}\left\{f_{a_{1} b_{1}}{ }^{(T)}(\lambda), f_{a_{2} b_{2}}{ }^{(T)}(\lambda)\right\}=2 \pi P^{4}\left\{G_{a_{1} b_{1}}{ }^{(T)}(0) G_{a_{2} b_{2}}{ }^{(T)}(0)\right\}^{-1} \times \\
\times G_{a_{1} a_{2} b_{1} b_{2}}(0)\left[\int_{t_{1}=0}^{T} \Psi_{a_{1} b_{1}}{ }^{(T)}(\alpha) \Psi_{a_{2} b_{2}}{ }^{(T)}\left(\lambda_{2}-\lambda_{1}+\alpha\right) V Z d t_{1}+\right. \\
\left.+\int_{t_{1}=0}^{T} \Psi_{a_{1} b_{1}}{ }^{(T)}(\alpha) \Psi_{a_{2} b_{2}}{ }^{(T)}\left(\frac{\lambda_{2}+\lambda_{1}}{B_{T}}-\alpha\right) V Z d t_{1}\right]+O\left(T^{-2}\right)
\end{gathered}
$$

when $B_{T}=1$

$$
\begin{gathered}
\operatorname{cov}\left\{f_{a_{1} b_{1}}{ }^{(T)}(\lambda), f_{a_{2} b_{2}}{ }^{(T)}(\lambda)\right\}= \\
=\left\{\int_{t_{1}=0}^{T} \Psi_{a_{1} b_{1}}{ }^{(T)}(\alpha) \Psi_{a_{2} b_{2}}{ }^{(T)}(\alpha) d t_{1}\right\}\left[\delta\left(\lambda_{1}-\lambda_{2}\right) V Z+\delta\left(\lambda_{1}+\lambda_{2}\right) V Z\right]+ \\
\left.+\int_{t_{1}=0}^{T} \Psi_{a_{1} b_{1}}{ }^{(T)}(\alpha) \Psi_{a_{2} b_{2}}{ }^{(T)}\left(\frac{\lambda_{2}+\lambda_{1}}{B_{T}}-\alpha\right) V Z d t_{1}\right]+O\left(B_{T}^{-1} T^{-2}\right),
\end{gathered}
$$

when $B_{T}^{-1} \rightarrow 0 \quad B_{T} T \rightarrow \infty$ as $T \rightarrow \infty$.

\section{Corollary 3.3}

Under the conditions of Theorem (3.2) if $\lambda \neq 0, \lambda \in R$ and $B_{T} \rightarrow 0$ as $T \rightarrow \infty$, then

$$
E\left\{f_{a b}^{(T)}(\lambda)\right\}_{T \rightarrow \infty}^{\rightarrow} P^{2}\left[\begin{array}{cc}
f_{a_{1} a_{2}}(\alpha) & f_{a_{1} b_{2}}(\alpha) A(\alpha)^{T} \\
A(\alpha) f_{b_{1} a_{2}}(\alpha) & A(\alpha) f_{b_{1} b_{2}}(\alpha) A(\alpha)^{T}
\end{array}\right]
$$

\section{Proof}

proof comes directly by taking the limits for both sides of formula (3.5) as $T \rightarrow \infty$

\section{Corollary 4.2.}

Under the conditions of theorem (3.2) if the spectral density function $f_{a b}(x)$ is bounded by a constant $M$, $a, b=1, \ldots, \min (r+s)$ and continuous at a point $x=\lambda, \lambda \in R$ and $B_{T} \rightarrow 0, B_{T} T \rightarrow \infty$ as $T \rightarrow \infty$, then

$$
\operatorname{cov}\left\{f_{a_{1} b_{1}}{ }^{(T)}(\lambda), f_{a_{2} b_{2}}{ }^{(T)}(\lambda)\right\} \underset{T \rightarrow \infty}{\longrightarrow} 0
$$

for all $a_{j}, b_{j}=1, \ldots, \min (r+s), \lambda_{j} \in R, j=1, \ldots ., k, k=1,2, \ldots$

\section{Proof}

Taking the modulus for both sides of equation (3.6), then using Assumption III and the boundedness of $f_{a b}(\lambda)$ by constant $M$, we get

$$
\operatorname{cov}\left\{f_{a_{1} b_{1}}{ }^{(T)}(\lambda), f_{a_{2} b_{2}}{ }^{(T)}(\lambda)\right\}=O\left(B_{T}^{-1}\right)=O\left(B_{T}^{-1} T^{-1}\right) \underset{T \rightarrow \infty}{\longrightarrow} 0 .
$$

Then the corollary is obtained . 


\section{Application on the Theoretical Study:}

We will apply our theoretical case study in climate as following :

\subsection{Studying the Atmospheric Pressure and Maximum temperature.}

The data in this research represents the daily maximum temperatures and atmospheric pressure in Tripoli for the period from 1/1/ 2016 to 29/2/2016.

\subsubsection{Studying the Atmospheric pressure.}

In this study we will comparison between our results, model of strictly stability time series (the Atmospheric pressure) with some missing observations and the classical results, where all observations are available.

Let $X_{a}(t)$ is the data of the daily of the atmospheric pressure where all observations are available (classical case) suppose that there is some missing observations in a random way (our study), table 4.1.1 shows the comparison between our results, spectral analysis of strictly stability time series with some missing observations and the classic results, where all observations are available.

Table 4.1.1:- comparison of the results with and without missed observations of the Atmospheric pressure

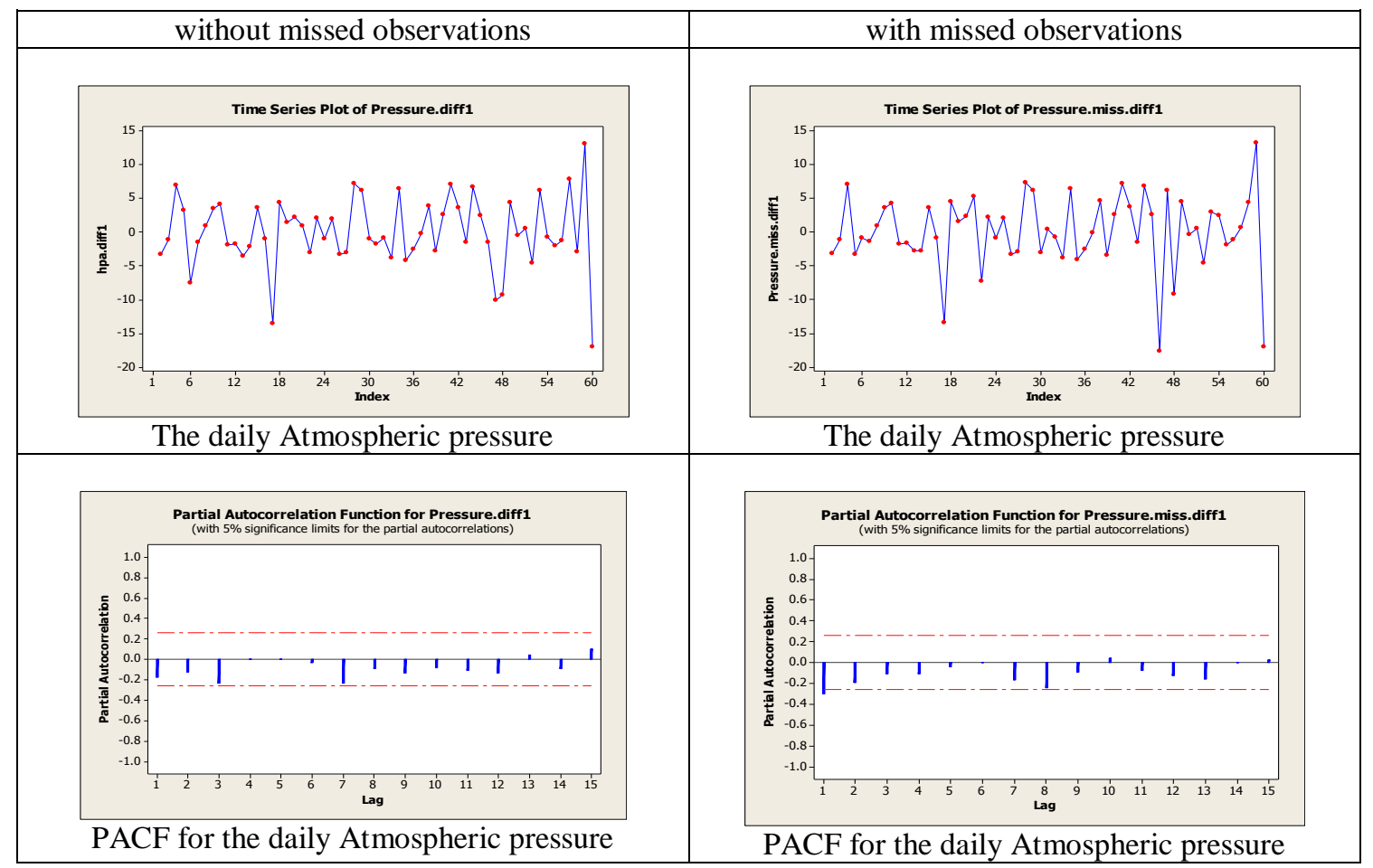




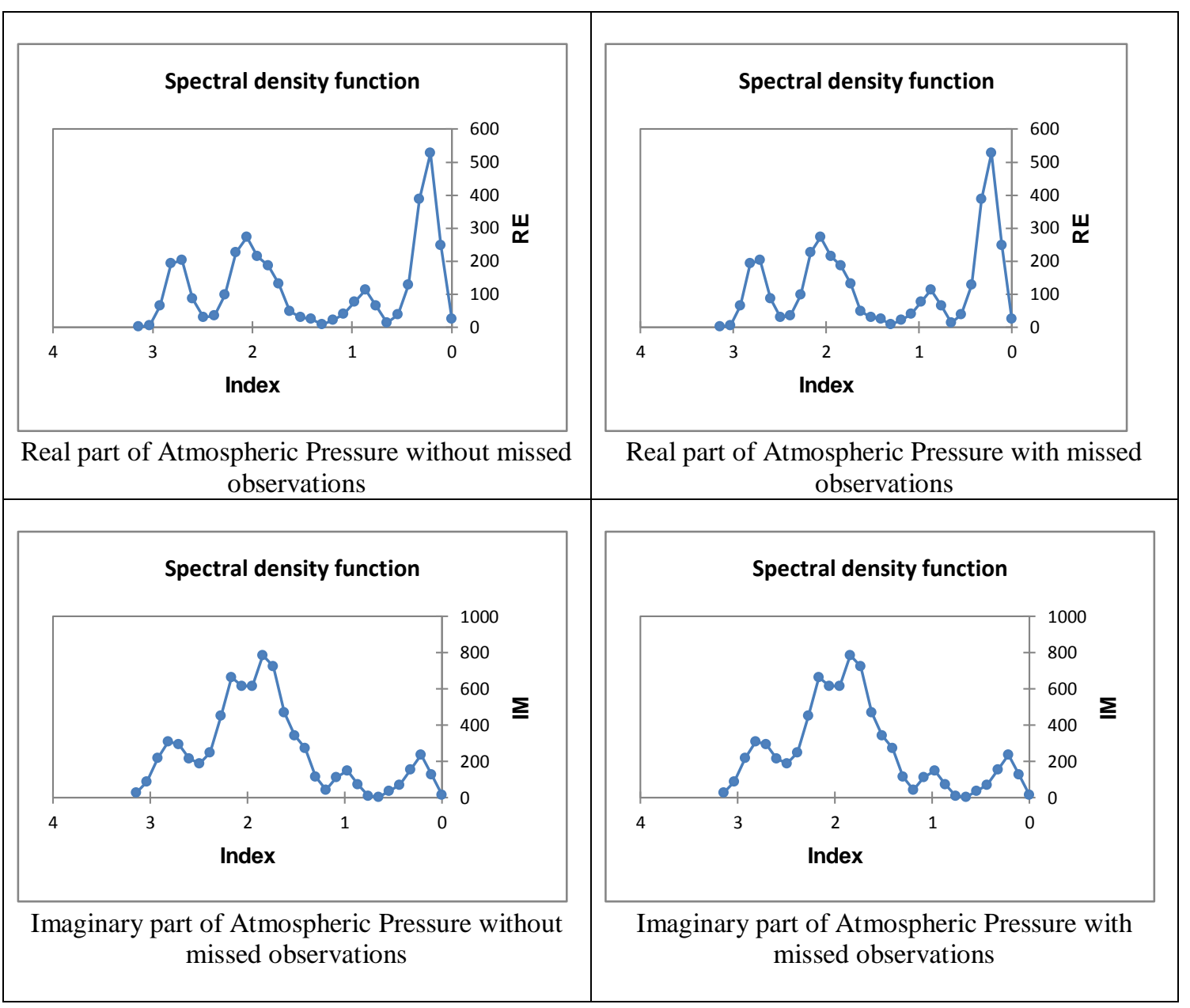




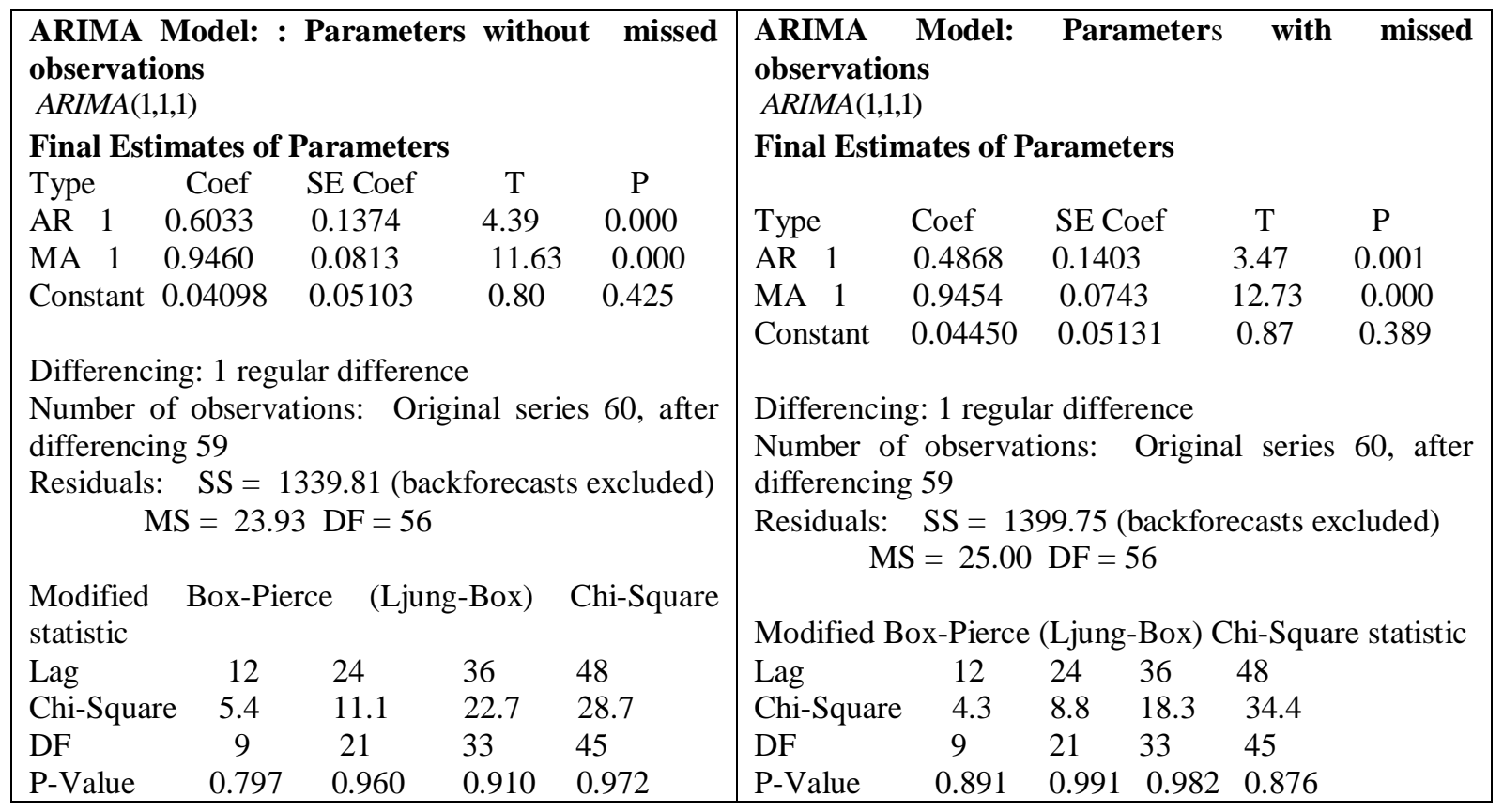

4.1.2. Studying the Maximum temperature:-

In this study we will comparison between our results, model of strictly stability time series (the Maximum temperature) with some missing observations and the classical results, where all observations are available.

Let $Y_{a}(t)$ is the data of the daily of the Maximum temperature where all observations are available (classical case) suppose that there is some missing observations in a random way (our study), table 4.1.2 shows the comparison between our results, spectral analysis of strictly stability time series with some missing observations and the classic results, where all observations are available.

Table (4.1.2) comparison of the results with and without missed observations of the Maximum temperature.

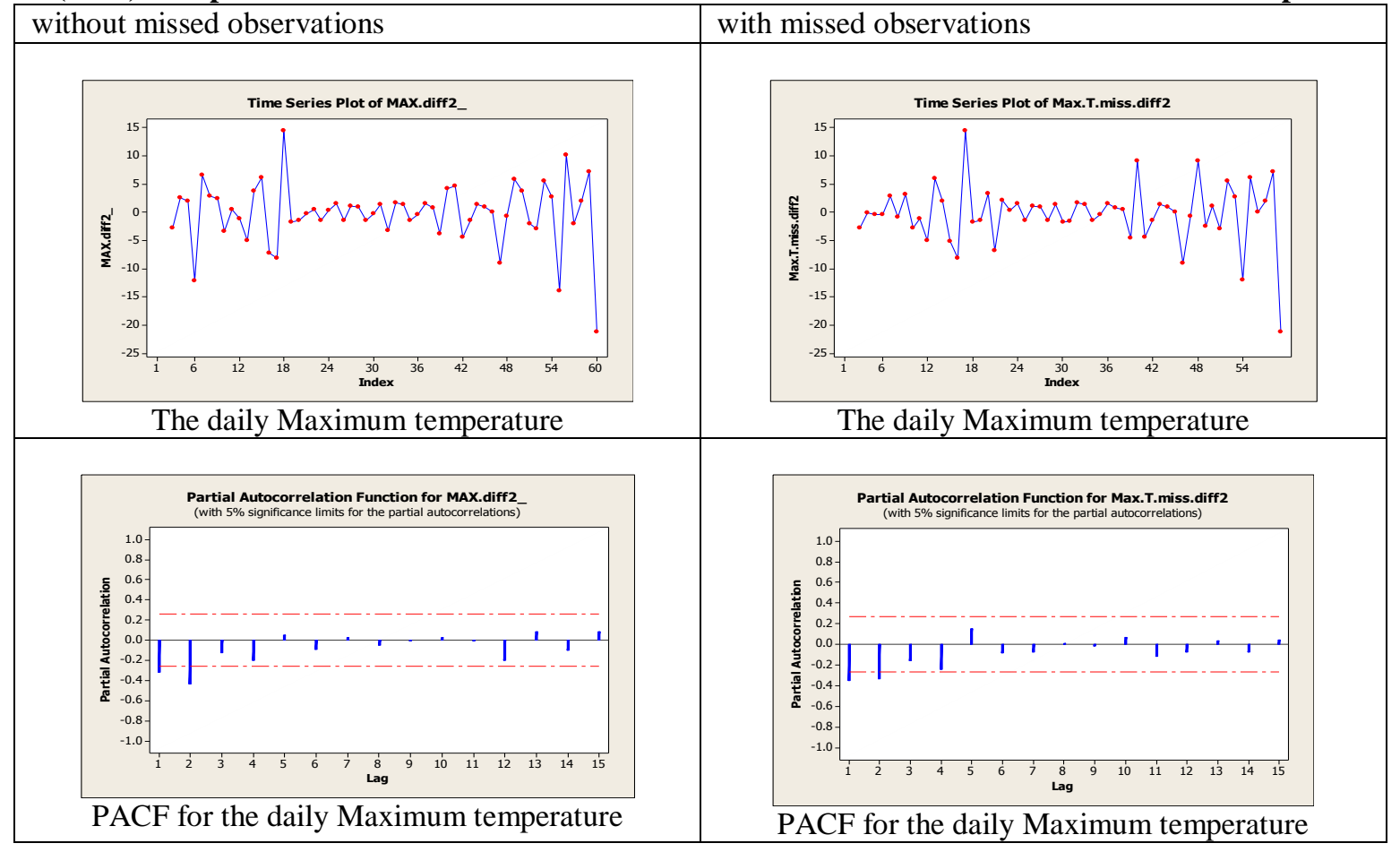




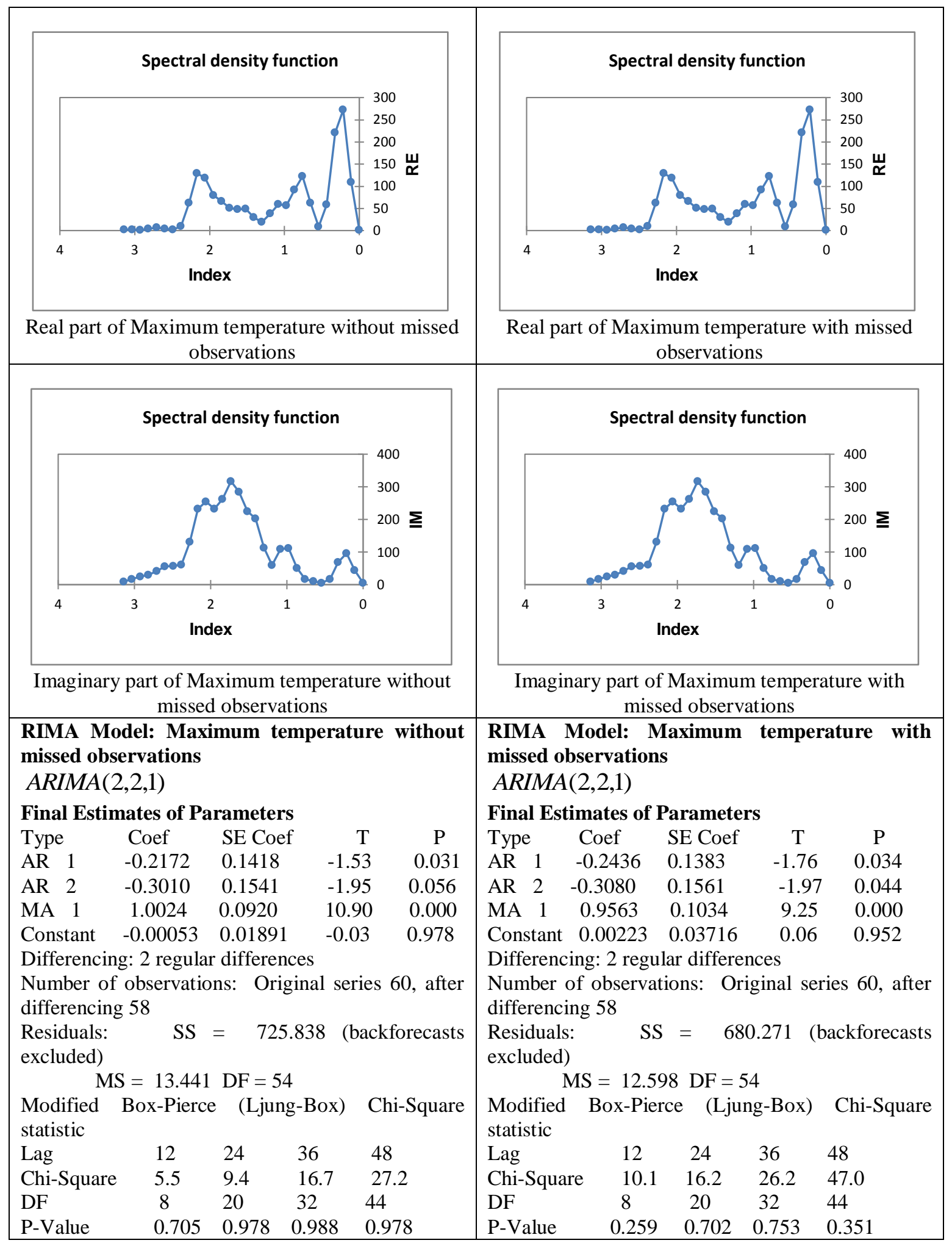

4.1.3. Studying the Regression Between the Atmospheric pressure and the Maximum temperature In this study we will comparison between our results, regression model between the Atmospheric pressure and the Maximum temperature with some missing observations and the classical results, where all observations are available, the comparison between two cases is shown in table (4.1.3) . 
Table 4.1.3:- Comparison of the results with and without missed observations of the regression analysis

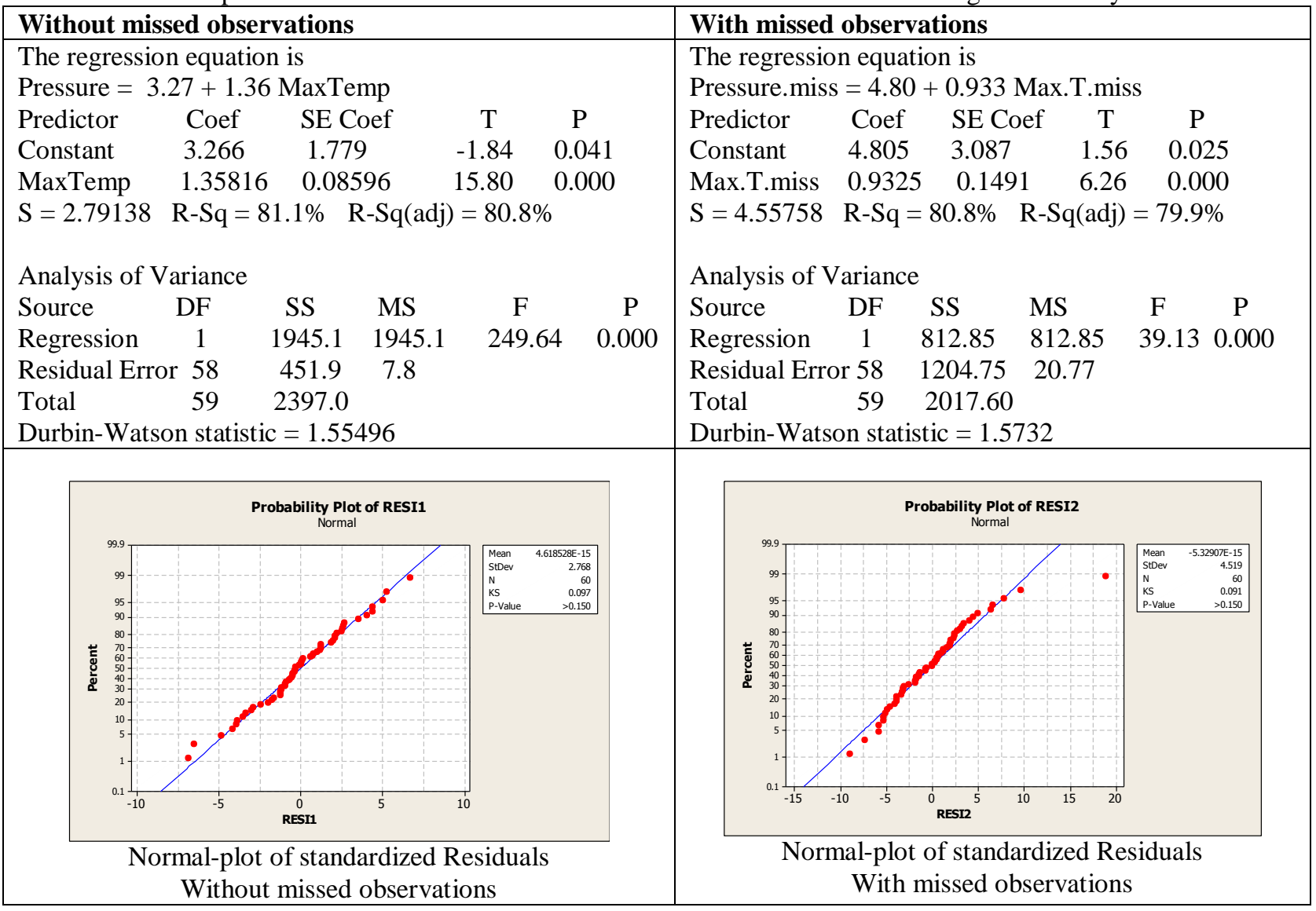

Materials and Methods:-

We used SPSS and MINITAB, XL.STAT the software programming to solve our numerical example .

\section{Results and Discussion:-}

1. The study of the time series with missed observations and with the modified periodogram had the same results as the study of the classical time series .

2. The study of regression model between classical time series $\mathrm{X}(\mathrm{t}), \mathrm{Y}(\mathrm{t})$ had the same results as case of missed observations .

\section{References:-}

1. D.R. Brillinger, M.Rosenblatt, Asymptotic theory of estimates of k-th order spectra, in: B. Harris (Ed.), Advanced Seminar on Spectral Analysis of time series, Wiley, New York, 1967, pp.153-188 .

2. D.R. Brillinger, "Asymptotic properties of spectral estimates of second order", Biometrika56(2), (1969)375390.

3. R. Dahlhaus, "On a spectral density estimate obtained by averaging periodograms", J. Appl. Probab. 22(1985) 592-610.

4. (4) M.A. Ghazal, E.A. Farag, "Estimating the spectral density, autocovariance function and spectral measure of continuous-time stationary processes, in: Annual Conference", ISSR, Cairo, Vol.33, no. part, 1998, pp.1-20.

5. M.A. Ghazal, "On a spectral density estimate on non-crossed intervals observation", Int. J. Appl. Math.1 (8) (1999) 875-882.

6. M.A. Ghazal, E.A. Farag, "Some Properties of The Expanded Finite Fourier Transform, Statistica", anno LX, no.3, 2000.

7. D.R. Brillinger, Time Series Data Analysis and Theory (2001).

8. A.A.M. Teamah , H.S. Bakouch, " Multitaper Multivriate Spectral Estimators Time Series with Distorted Observations", International Journal of Pure and Applied Mathematics,Vol,No.1(2004),45-57. 
9. Chris Chatfiold, The Analysis of Time series An Introduction .

10. M. A. Ghazal, E. A. Farag, A.E EL-Desokey," Some properties of the Discrete Expanded finite Fourier transform with missed Observations", Vol.40, No.3, 30 September (2005), pp887-902.

11. M.A. Ghazal and A. Elhassanein, "Periodogram Analysis with Missing Observations", J.Appl. Math. Computing Vol.22 (2006), No.1-2,pp209-222.

12. M.A. Ghazal, G.S. Mokaddis, A.E. El-Desokey, "Spectral analysis of strictly stationary continuous time series", Journal of Mathematical Sciences, Vol.3, No.1 (2009).

13. G.S. Mokaddis, M.A. Ghazal and A.E. El-Desokey, " Asymptotic Properties of Spectral Estimates of SecondOrder with Missed Observations", Journal of Mathematics and statistics 6(1):10-16,(2010).

14. A. ELhassanein, "Nonparametric Spectral Analysis On Disjoint Segments Of Observations", Journal of the Applied Mathematics, Statistics and Informatics(JAMSI), 7 (2011),No.1.

15. A. ELhassanein, "On the Theory of continuous Time series", Indian J. Pure Appl .Math, 45 (3): 297-310, June (2014).

16. M.A.Ghazal, A.I.El-desokey, A.M.Ben Aros "Statistical analysis of linear stability continuous time seies between two vector valued stochastic process" International Journal of Scientific Engineering Research, Volume8,Issue4,April-2017 ISSN 2229-5518.

17. M.A. Ghazal, A.I. El-Desokey, M.A. Alargt " Asymptotic properties of the discrete stability time series with missed observations between two-vector valued stochastic process" International Research Journal of Engineering and Technology (IRJET) Volume4,Issue4,April-2017. 